\title{
DIFFERENCE EQUATIONS OVER LOCALLY COMPACT ABELIAN GROUPS \\ BY
}

\author{
G. A. EDGAR AND J. M. ROSENBLATT
}

\begin{abstract}
A homogeneous linear difference equation with constant coefficients over a locally compact abelian group $G$ is an equation of the form $\sum_{j=1}^{n} c_{j} f\left(t_{j} x\right)=0$ which holds for all $x \in G$ where $c_{1}, \ldots, c_{n}$ are nonzero complex scalars, $t_{1}, \ldots, t_{n}$ are distinct elements of $G$, and $f$ is a complexvalued function on $G$. A function $f$ has linearly independent translates precisely when it does not satisfy any nontrivial linear difference equation. The locally compact abelian groups without nontrivial compact subgroups are exactly the locally compact abelian groups such that all nonzero $f \in$ $L_{p}(G)$ with $1<p<2$ have linearly independent translates. Moreover, if $G$ is the real line or, more generally, if $G$ is $R^{n}$ and the difference equation has a characteristic trigonometric polynomial with a locally linear zero set, then the difference equation has no nonzero solutions in $C_{0}(G)$ and no nonzero solutions in $L_{p}(G)$ for $1<p<\infty$. But if $G$ is some $R^{n}$ for $n>2$ and the difference equation has a characteristic trigonometric polynomial with a curvilinear portion of its zero set, then there will be nonzero $C_{0}\left(R^{n}\right)$ solutions and even nonzero $L_{p}\left(R^{n}\right)$ solutions for $p>2 n /(n-1)$. These examples are the best possible because if $1<p<2 n /(n-1)$, then any nonzero function in $L_{p}\left(R^{n}\right)$ has linearly independent translates. Also, the solutions to linear difference equations over the circle group can be simply described in a fashion which an example shows cannot be extended to all compact abelian groups.
\end{abstract}

0. Introduction. A homogeneous linear difference equation with constant coefficients in the complex numbers $C$ over the group of integers $Z$ is an equation of the form $\sum_{j=1}^{n} c_{j} f\left(z_{j}+z\right)=0$ which holds for all $z \in Z$ where $c_{1}, \ldots, c_{n} \in C$ are nonzero, the elements $z_{1}, \ldots, z_{n} \in Z$ are distinct, and $f$ : $Z \rightarrow C$. It is well known (see [1] and [9, p. 163]) that the only solutions of such difference equations are of the form $f(z)=\sum_{k=1}^{K} Q_{k}(z) a_{k}^{z}$ where the $Q_{k}$ are polynomials over $C$ and the scalars $a_{k} \in C$ are nonzero. If one assumes in addition that $|f(z)| \leqslant B|z|^{E}$ for some constants $B, E>0$, then each $a_{k}$ has modulus one; if $f$ is bounded, then also each $Q_{k}$ is a constant polynomial. Hence, the only functions in $l_{\infty}(Z)$ which satisfy a nontrivial difference equation over $Z$ are the trigonometric polynomials on $Z$, linear combinations over $C$ of complex characters on $Z$. As a consequence, the only function in

Presented to the Society, June 16, 1978; received by the editors September 5, 1978. AMS (MOS) subject classifications (1970). Primary 42A68, 43A25, 39A10. 
$c_{0}(Z)$ or $l_{p}(Z)$ with $1 \leqslant p<\infty$ that has a dependence relation among its translates is the zero function.

To generalize the concept of a difference equation to a locally compact abelian group $G$, choose nonzero elements $c_{1}, \ldots, c_{n} \in C$ and distinct elements $t_{1}, \ldots, t_{n} \in G$. For $f: G \rightarrow C$, define the difference operator $D$ by $D f=\sum_{j=1}^{n} c_{j} T_{t j} f$ where the translation operators $T_{t j}$ are defined by $T_{t} f(x)=$ $f(t x)$ for any $t, x \in G$. A difference equation is then any equation of the form $D f=0$. One of the major problems in difference equations is to describe all solutions $f: G \rightarrow C$ to a given difference equation $D f=0$; one would like a form for the solutions which makes it clear which measurable functions in some $L_{p}(G)$ with $1 \leqslant p \leqslant \infty$ can satisfy a difference equation. If there are any solutions to the difference equation at all, there will be solutions in $L_{\infty}(G)$; but there may be no solutions in $L_{p}(G)$ with $1 \leqslant p<\infty$ or in $C_{0}(G)$ depending on the nature of the group and the difference equation. For background and notation see [5], [6], [23], [24] and [29].

1. General locally compact abelian groups. Suppose $D f=\sum_{j=1}^{n} c_{j} T_{i} f$ is a difference operator on a locally compact abelian group $G$ and $f \in L_{1}(G)$. Then the Fourier transform $\hat{f}$ is defined on the dual group $\hat{G}$ by

$$
\hat{f}(\gamma)=\int_{G} f(s) \overline{\gamma(s)} d \lambda_{G}(s) \text { for all } \gamma \in \hat{G},
$$

where $\lambda_{G}$ is a fixed Haar measure in $G$. The Fourier transform of $D f \in L_{1}(G)$ is also well defined and, for all $\gamma \in \hat{G}$,

$$
(D f)^{\wedge}(\gamma)=\hat{f}(\gamma) \sum_{j=1}^{n} c_{j} \gamma\left(t_{j}\right)
$$

Since $\hat{f} \in C_{0}(\hat{G})$, the only way that one can have $D f=0$ a.e. $\left[\lambda_{G}\right]$ is to have $f=0$ a.e. $\left[\lambda_{G}\right]$ or to have the sum $\sum_{j=1}^{n} c_{j} \gamma\left(t_{j}\right)$ vanishing for $\gamma$ in a nonempty open subset of $\hat{G}$. Therefore, to see whether $D f=0$ is possible with $0 \neq f \in$ $L_{1}(G)$, one must consider the zero set $\mathscr{Z}_{p}=\{\gamma \in \hat{G}: p(\gamma)=0\}$ of the trigonometric polynomial $p: \hat{G} \rightarrow C$ defined by $p(\gamma)=\sum_{j=1}^{n} c_{j} \gamma\left(t_{j}\right)$. This polynomial is called the characteristic trigonometric polynomial of the difference equation. Although the following lemma is known (see Ross [27], [28] and López and Ross [21, p. 123]), the argument given here seems to be new and somewhat simpler than the ones given previously.

LEMMA 1.1. Suppose $G$ is a locally compact abelian group without nontrivial compact subgroups. Then a trigonometric polynomial on $\hat{G}$ which vanishes in a set of positive measure with respect to $\lambda_{\hat{G}}$ must vanish on all of $\hat{G}$.

Proof. The assumption on $G$ is equivalent to assuming $\hat{G}$ is connected (see Hewitt and Ross [11, p. 383]). Therefore, assume $X$ is a connected locally compact abelian group and $p$ is a trigonometric polynomial on $X$ which 
vanishes on a compact set $K \subset X$ with $\lambda_{X}(K)>0$.

First, consider the function

$$
F(x)=\lambda_{X}\left(K \cap K x^{-1} \cap \cdots \cap K x^{-n}\right) .
$$

This is a continuous function of $x$ and $F(1)=\lambda_{X}(K)>0$. Hence, there exists an open neighborhood $U$ of 1 such that $F(u)>0$ for all $u \in U$. But then for all $u \in U$, there exists $y \in K$ such that $y u^{l} \in K$ for $l=1, \ldots, n$. Compare with Kemperman [16].

Now let $\gamma_{1}, \ldots, \gamma_{n}$ be distinct characters on $X$ and let $c_{1}, \ldots, c_{n}$ be complex numbers with $p(x)=\sum_{k=1}^{n} c_{k} \gamma_{k}(x)$. For $k_{1}, k_{2}=1, \ldots, n$, let

$$
E_{k_{1} k_{2}}=\left\{x \in X: \gamma_{k_{1}}(x)=\gamma_{k_{2}}(x)\right\} \text {; }
$$

the set $E_{k_{1} k_{2}}$ is a closed subgroup and, if $k_{1} \neq k_{2}$, it contains no interior. Indeed, if $E_{k_{1} k_{2}}$ contains interior, then it is open and closed. Since $X$ is connected, this implies $X=E_{k_{1} k_{2}}$ and $k_{1}=k_{2}$. Hence the set

$$
U \backslash \bigcup_{k_{1} \neq k_{2}}\left\{x \in X: \gamma_{k_{1}}(x)=\gamma_{k_{2}}(x)\right\}
$$

is an open dense subset of $U$.

Thus, there exists $u \in U$ such that $\left\{\gamma_{k}(u): k=1, \ldots, n\right\}$ are distinct complex numbers. Choose $y \in K$ such that $y u^{l} \in K$ for $l=1, \ldots, n$. Then

$$
0=p\left(y u^{\prime}\right)=\sum_{k=1}^{n} c_{k} \gamma_{k}(y) \gamma_{k}(u)^{l}
$$

for $l=1, \ldots, n$. But since $\left\{\gamma_{k}(u): k=1, \ldots, n\right\}$ are distinct and the Vandermonde determinant $\operatorname{det}\left(\gamma_{k}(u)^{l}\right) \neq 0$, we must have $c_{k} \gamma_{k}(y)=0$ for all $k=1, \ldots, n$. So $c_{k}=0$ for all $k=1, \ldots, n$ and $p=0$.

A nontrivial difference operator $D$ cannot be identically zero on $L_{1}(G)$ and so its characteristic trigonometric polynomial cannot vanish identically on $\hat{G}$. Hence, Lemma 1.1 proves that in a locally compact abelian group without nontrivial compact subgroups, no nonzero $f \in L_{1}(G)$ can be a solution to a nontrivial difference equation. Conversely, if $K$ is any compact subgroup of $G$, there exists a compact neighborhood $K_{0} \subset G$ with $k K_{0}=K_{0}$ for all $k \in K$; hence, if $G$ contains a nontrivial compact subgroup, then there exists a nonzero characteristic function $f \in L_{1}(G)$ and some $k \neq 1$ in $G$ with $T_{k} f-f=0$. In addition, because the Fourier transform is also well-defined and one-to-one from $L_{p}(G) \rightarrow L_{p /(p-1)}(\hat{G})$ when $1<p \leqslant 2$, the following theorem is a consequence of Lemma 1.1.

THEOREM 1.2. Suppose $G$ is a locally compact abelian group without nontrivial compact subgroups. Then any nonzero function in $L_{p}(G)$ with $1 \leqslant p \leqslant 2$ has linearly independent translates.

The examples in $\$ 2$ will show why the restriction on $p$ is necessary even in 
Euclidean spaces, but there are other classes of functions on locally compact abelian groups without nontrivial compact subgroups that will also have linearly independent translates.

LEMMA 1.3. Suppose $G$ is a locally compact abelian group without nontrivial compact subgroups and let $E$ be a nonempty subset of a compact subset of $G$. Then for distinct elements $g_{1}, \ldots, g_{n} \in G$, there always exists some $i=$ $1, \ldots, n$ such that $g_{i} E \backslash \cup\left\{g_{j} E: j \neq i\right\} \neq \varnothing$.

Proof. One of the other characterizations of a locally compact abelian group $G$ with connected dual group is that for $g \in G, g \neq 1$, there exists a continuous homomorphism $\varphi: G \rightarrow R$ with $\varphi(g) \neq 0$ (see Hewitt and Ross $\left[11\right.$, p. 390]). For each $i \neq j$, choose a continuous homomorphism $\varphi_{i j}: G \rightarrow R$ with $\varphi_{i j}\left(g_{i}\right) \neq \varphi_{i j}\left(g_{j}\right)$. With $d=n(n-1) / 2$, define $\varphi: G \rightarrow R^{d}$ by

$$
\varphi(g)=\left(\varphi_{i j}(g): i, j=1, \ldots, n, i \neq j\right)
$$

where some fixed order for the indices is chosen independently of $g \in G$. Then $\varphi$ is a continuous homomorphism of $G$ into $R^{d}$ with $\varphi\left(g_{1}\right), \ldots, \varphi\left(g_{n}\right)$ distinct and $\varphi(E)$ contained in some compact set. If some $\varphi\left(g_{i}\right) \varphi(E)$ is not contained in the union of the other $\varphi\left(g_{j}\right) \varphi(E)$, then the same is true of $g_{i} E$. Hence, without loss of generality, $G$ is $R^{d}$. But then it is an obvious geometrical fact that for distinct vectors $g_{1}, \ldots, g_{n} \in R^{d}$, there is a vector $g_{0} \in R^{d}$ such that the scalar products $g_{0} \cdot g_{i}, i=1, \ldots, n$, are distinct. That is, by taking another continuous homomorphism $\Psi: R^{d} \rightarrow R$ given by $\Psi(g)$ $=g_{0} \cdot g$, the lemma is reduced to the case that $G$ is $R$ itself. But in the real line, if the elements $g_{1}, \ldots, g_{n}$ are distinct, then some one $g_{i}$ must be largest and for this $g_{i}, g_{i} E$ is not contained in the union of the other $g_{j} E$.

REMARK. The proof shows that there are always two elements $g_{i_{1}} E$ and $g_{i_{2}} E$ which are not contained in the union of the other $g_{j} E$.

THEOREM 1.4. Suppose $G$ is a locally compact abelian group without nontrivial compact subgroups. Let $f \neq 0$ be any of the following:

(1) a function which vanishes outside a compact set,

(2) an element in $L_{\infty}(G)$ which vanishes outside a compact set,

(3) a measure with compact support,

(4) a distribution with compact support.

Then $f$ has linearly independent translates.

Proof. In case (1), let $E=\{x \in G: f(x) \neq 0\}$; then suppose some distinct elements $g_{1}, \ldots, g_{n} \in G$, and scalars $c_{1}, \ldots, c_{n} \in C$ satisfy $\sum_{j=1}^{n} c_{j} T_{t} f=0$. Using Lemma 1.3 with this $E$ and $g_{1}^{-1}, \ldots, g_{n}^{-1}$, we can find some $i=$ $1, \ldots, n$ and some point $x \in g_{i}^{-1} E$ with $x \notin g_{j}^{-1} E$ for all $j \neq i$. So

$$
0=\sum_{j=1}^{n} c_{j} f\left(g_{j} x\right)=c_{i} f\left(g_{i} x\right)
$$


while $f\left(g_{i} x\right) \neq 0$. Hence $c_{i}=0$ and $\sum_{j \neq i} c_{j} T_{g_{f}} f=0$; by induction, all the coefficients $c_{j}=0$. In case (2), the argument is the same except that a lifting which commutes with the group action must be used because one would only begin with the assumption that $\sum_{j=1}^{n} c_{j} T_{t} f=0$ a.e. $\left[\lambda_{G}\right]$; see [12] for the existence and relevant properties of such a lifting. The cases (3) and (4) are treated similarly.

We make one further remark about the general locally compact abelian group. Whenever the zero set of the characteristic polynomial is nonempty, there will be characters which satisfy the difference equation; other functions in $L_{\infty}(G)$ or distributions which satisfy the difference equation can then be obtained as a distributionally convergent sum of those characters which are solutions. However, the theorems in Jenkins [13] and in Rosenblatt [26] do establish the following limitation.

THEOREM 1.5. A nonzero positive bounded function on a locally compact abelian group can satisfy a difference equation $\sum_{j=1}^{n} c_{j} T_{g} f=0$ only if $\sum_{j=1}^{n} c_{j}=$ 0 .

2. The groups $R^{n}$. For some locally compact abelian groups, Theorem 1.2 is not the best possible one of its kind. If the group $G$ is the integers, then the classical solution of a homogeneous difference equation with constant coefficients shows that no restriction on the $L_{p}$-space other than $0<p<\infty$ needs to be made; indeed, any nonzero function in $c_{0}(Z)$ has linearly independent translates. This fact is really a special case of Theorem 2.9 which will simultaneously prove that any nonzero function in $C_{0}(R)$ or in $L_{p}(R)$ with $1 \leqslant p<\infty$ has linearly independent translates. However, one cannot extend Theorem 1.2 to include $C_{0}(G)$ or all $L_{p}(G)$ with $p>2$ in general as the following example shows. This example is really a special case of much deeper theorems that are already known and which will be discussed subsequently; we present it here to stress the truly elementary nature of the phenomenon.

EXAMPLE 2.1. Consider the difference equation over $\boldsymbol{R}^{2}$ :

$$
2 f(x, y)=f(x+1, y)+f(x-1, y)+f(x, y+1)+f(x, y-1) .
$$

If one takes the Fourier transform of

$$
D f=2 f-T_{(1,0)} f-T_{(-1,0)} f-T_{(0,1)} f-T_{(0,-1)} f,
$$

then one sees that the characteristic trigonometric polynomial is $p(x, y)=2(1$ $-\cos x-\cos y$ ). This difference equation was chosen with the fact in mind that $\Psi_{p}$ contains curvilinear arcs. With $\cos ^{-1}:[0,1] \rightarrow[0, \pi / 2]$ denoting the inverse cosine function, the curve

$$
c(t)=\left(\cos ^{-1}(t), \cos ^{-1}(1-t)\right) \text { for } t \in[0,1]
$$


has an image contained in $\mathscr{Z}_{p}$. This proves that the function

$$
f(x, y)=\int_{1 / 3}^{2 / 3} \exp (i(x, y) \cdot c(t)) d t
$$

satisfies the difference equation above, although a direct substitution will do the same. The function $f$ is continuous and, as the arguments which follow show, satisfies an estimate $|f(x, y)| \leqslant A /(|x|+|y|)^{1 / 2}$ for a constant $A$ independent of $x$ and $y$. This function then is in $C_{0}\left(R^{2}\right)$, in all $L_{p}\left(R^{2}\right)$ for $p>4$, and also has a dependence relation among its translates.

To prove the estimate on $|f(x, y)|$, one can use two lemmas of van der Corput which are standard tools for getting asymptotic estimates (see [17, pp. 15-16]).

LEMMA 2.2. Suppose $h$ is a real-valued function with a monotone derivative $h^{\prime}$ on $[a, b]$ with $h^{\prime}(t) \geqslant \lambda>0$ or $h^{\prime}(t) \leqslant-\lambda<0$ for all $t \in[a, b]$. Then the integral $J=\int_{a}^{b} \exp (2 \pi i h(t)) d t$ satisfies $|J| \leqslant 1 / \lambda$.

LEMMA 2.3. Suppose $h$ is a twice differentiable real-valued function on $[a, b]$ with $h^{\prime \prime}(t) \geqslant \rho>0$ or $h^{\prime \prime}(t) \leqslant-\rho<0$ for all $t \in[a, b]$. Then the integral $J$ of Lemma 2.2 satisfies $|J| \leqslant 4 / \sqrt{\rho}$.

These two lemmas apply to estimating $|f(x, y)|$ if we let $h(t)=$ $(1 / 2 \pi)(x, y) \cdot c(t)$. One computes

$$
h^{\prime}(t)=\frac{1}{2 \pi}\left(\frac{-x}{\left(1-t^{2}\right)^{1 / 2}}+\frac{y}{\left(1-(1-t)^{2}\right)^{1 / 2}}\right)
$$

and

$$
h^{\prime \prime}(t)=\frac{1}{2 \pi}\left[\frac{-x t}{\left(1-t^{2}\right)^{3 / 2}}+\left[\frac{-y(1-t)}{\left(1-(1-t)^{2}\right)^{3 / 2}}\right]\right] \text {. }
$$

If both $x$ and $y$ are positive, then $h^{\prime \prime}(t) \leqslant-B_{1}(x+y)$ for some constant $B_{1}>0$ independent of $x$ and $y$ and all $t \in\left[\frac{1}{3}, \frac{2}{3}\right]$. If both $x$ and $y$ are negative, then $h^{\prime \prime}(t) \geqslant-B_{1}(x+y)$ for all $t \in\left[\frac{1}{3}, \frac{2}{3}\right]$. Hence, Lemma 2.3 proves that in the first and third quadrants of $R^{2},|f(x, y)|<A_{1} /(|x|+$ $|y|)^{1 / 2}$ for some constant $A_{1}$ which is independent of $x$ and $y$.

To get an estimate for $|f(x, y)|$ in the second and fourth quadrants of $R^{2}$, one uses Lemma 2.2 because in these quadrants $h^{\prime \prime}$ may vanish. However, for any $(x, y)$, the function $h^{\prime \prime}(t)$ can be zero at most once on $\left[\frac{1}{3}, \frac{2}{3}\right]$ as a straightforward calculation shows, and so $h^{\prime}(t)$ is piecewise monotone in two pieces on $\left[\frac{1}{3}, \frac{2}{3}\right]$. Also, if $(x, y)$ is in the second quadrant, then there is a constant $B_{2}>0$ independent of $x$ and $y$ such that $h^{\prime}(t) \geqslant B_{2}(|x|+|y|)$ for all 
$t \in\left[\frac{1}{3}, \frac{2}{3}\right]$. If $(x, y)$ is in the fourth quadrant, then $h^{\prime}(t) \leqslant-B_{2}(|x|+|y|)$ for all $t \in\left[\frac{1}{3}, \frac{2}{3}\right]$. Hence, Lemma 2.2 proves that in the second and fourth quadrants of $R^{2},|f(x, y)| \leqslant A_{2} /(|x|+|y|)$ for some constant $A_{2}$ which is independent of $x$ and $y$. This together with the weaker estimate in the first and third quadrants gives $|f(x, y)| \leqslant A /(|x|+|y|)^{1 / 2}$ for some constant $A$ and all $(x, y) \in R^{2}$.

This one example is enough to show that for any $n \geqslant 2$, there are nonzero functions in $C_{0}\left(R^{n}\right)$ and in certain $L_{p}\left(R^{n}\right)$ with $2<p<\infty$ that do have a dependence relation among their translates. The essential idea is that when the zero set for a characteristic trigonometric polynomial of a difference equation over $R^{2}$ contains a nonlinear arc, then there will be $C_{0}\left(R^{2}\right)$ solutions to the difference equation. Indeed, suppose that the zero set contains a $C^{3}$-curve $c:[0,1] \rightarrow R^{2}$ such that for all nonempty open intervals $I \subset[0,1]$, $c(I)$ is not contained in a straight line in $R^{2}$. Then, by restricting the domain of $c$ and making a suitable quadratic change of variables, one can replace $c$ by a curve $c_{0}:[0,1] \rightarrow R^{2}$ with image also in the zero set such that, with $c_{0}(t)=(\alpha(t), \beta(t))$; one has (1) $\left|\alpha^{\prime}\right|,\left|\beta^{\prime}\right|,\left|\alpha^{\prime \prime}\right|,\left|\beta^{\prime \prime}\right|$ are bounded away from 0 , (2) either $\alpha^{\prime} \beta^{\prime}>0$ and $\alpha^{\prime \prime} \beta^{\prime \prime}<0$ or $\alpha^{\prime} \beta^{\prime}<0$ and $\alpha^{\prime \prime} \beta^{\prime \prime}>0$, and (3) $\alpha^{\prime \prime} / \beta^{\prime \prime}$ assumes any value at most once. One can then prove, using Lemmas 2.2 and 2.3 , that the function

$$
f(x, y)=\int_{0}^{1} \exp \left(i(x, y) \cdot c_{0}(t)\right) d t
$$

satisfies the difference equation in question and is a $C_{0}\left(R^{2}\right)$ function with $|f(x, y)| \leqslant A /(|x|+|y|)^{1 / 2}$ for some constant $A$. Hence, the explicit example above is really typical of difference equation over $R^{2}$ with a curvilinear section in the zero set of its characteristic trigonometric polynomial.

The evaluation of the asymptotic behavior of Fourier integrals of the type above is a difficult subject with a long history, only a small part of which is mentioned here. Suppose

$$
f(x, y)=\int_{\alpha}^{\beta} \exp (i(x, y) \cdot c(t)) d t
$$

where $c(t)$ is a $C^{2}$-curve in $R^{2}$; assume that $(x, y)$ is perpendicular to $c^{\prime}\left(t_{0}\right)$ for some unique point $t_{0} \in(\alpha, \beta)$, but that $(x, y) \cdot c^{\prime \prime}\left(t_{0}\right) \neq 0$. This is the situation in Example 2.1 for a cone of vectors $(x, y)$ lying in the first and third quadrants. One of the theorems of asymptotic expansions (see Erdélyi [7, p. 51]) shows that if $(x, y)=r(\cos \theta, \sin \theta)$, then as $r \rightarrow \infty$,

$$
f(x, y) \sim C r^{-1 / 2} \exp \left(i(x, y) \cdot c\left(t_{0}\right)+i \pi / 4\right)
$$

where the constant $C=2 \pi /(\cos \theta, \sin \theta) \cdot c^{\prime \prime}\left(t_{0}\right)$. Hence, the order $(|x|+$ $|y|)^{-1 / 2}$ is the correct order at infinity for $|f(x, y)|$. Because in Example 2.1 there is a cone of vectors $(x, y)$ in the first quadrant in which $(\cos \theta, \sin \theta)$. 
$c^{\prime \prime}(t)$ is bounded away from zero, the complex-valued function $f(x, y)$ of Example 2.1 is only in $L_{p}\left(R^{2}\right)$ for $p>4$. Although this resolves the question of the $L_{p}$-spaces to which this example belongs, it does not say whether there can be other solutions in $L_{p}$-classes for $p \leqslant 4$. In Corollary 2.7 , it is shown that the only solutions to this difference equation are in $L_{p}\left(R^{2}\right)$ for $p \geqslant 4$.

However, if one is willing to increase the dimension of the Euclidean space in which the difference equation is defined, then solutions in $L_{p}$-spaces with $p$ closer to 2 do certainly exist. Suppose $S$ is a compact $(n-1)$-dimensional surface in $R^{n}$, possibly with boundary, and $\mu$ is a smooth mass density on the surface, vanishing near the boundary. Let

$$
f(y)=\int_{S} \exp (i y \cdot x) \mu(x) d S(x)
$$

where $d S$ is the $(n-1)$-dimensional surface volume measure. Littman [20] proves a theorem which strengthens one due to Hlawka and Herz (see Herz [10]) that when $S$ is sufficiently smooth and at each point of $S$ some $k$ of the $n-1$ principal curvatures are different from zero, then $|f(y)| \leqslant A\left(\|y\|^{-k / 2}\right)$ for some constant $A$. This theorem is closely related to the asymptotic expansions which are studied in Erdélyi [7] and Chako [2], but there is a uniformity for the direction of $y$. These estimates may be used to give interesting examples of solutions to difference equations in $R^{n}$ with $n$ large.

EXAMPLE 2.4. Consider the difference equation in $R^{n}$ given by

$$
\begin{aligned}
2(n-1) f\left(x_{1}, \ldots, x_{n}\right) & =f\left(x_{1}+1, x_{2}, \ldots, x_{n}\right)+f\left(x_{1}-1, x_{2}, \ldots, x_{n}\right) \\
& +\cdots+f\left(x_{1}, \ldots, x_{n-1}, x_{n}+1\right)+f\left(x_{1}, \ldots, x_{n-1}, x_{n}-1\right) .
\end{aligned}
$$

The characteristic polynomial

$$
p\left(x_{1}, \ldots, x_{n}\right)=n-1-\sum_{j=1}^{n} \cos \left(x_{j}\right)
$$

has a zero set which is a disjoint union of compact $(n-1)$-dimensional surfaces of positive Gaussian curvature. Let $S_{n}$ be the connected component of the zero set containing the points with all coordinates zero except for one which is $\pm \pi / 2$; let $f_{n}$ be the Fourier transform of the surface volume element $d S_{n}$. Then by the theorem of Herz [10], $\left|f_{n}(y)\right| \leqslant A\left(\|y\|^{-(n-1) / 2}\right)$ for some constant $A$. This function $f_{n}$ satisfies the difference equation and $f_{n} \in C_{0}\left(R^{n}\right)$ $\cap L_{p}\left(R^{n}\right)$ for all $p$ with $p / 2>n /(n-1)$. Hence, for any $q>2$, there is a sufficiently large dimension $n$ for which there is some $f_{n} \in L_{q}\left(R^{n}\right)$ that has a dependence relation among its translates; this shows that Theorem 1.2 cannot be improved.

EXAMPLE 2.5. Consider any difference equation in $R^{2}$ for which there is a $C^{\infty}$-curve $c:[0,1] \rightarrow R^{2}$ whose image lies in the zero set of the characteristic 
trigonometric polynomial. The curvature of $c=(\alpha, \beta)$ is $\pm \mid \beta^{\prime} \alpha^{\prime \prime}-$ $\alpha^{\prime} \beta^{\prime \prime} \mid /\left(\left(\alpha^{\prime}\right)^{2}+\left(\beta^{\prime}\right)^{2}\right)^{3 / 2}$, which can only be zero on an open interval $I \subset$ $[0,1]$ if $c(I)$ is contained in a straight line. The nonlinearity of $c$ in Example 2.1 which gave the sign conditions on $\alpha$ and $\beta$ clearly forces the curvature to be nonvanishing. Hence, the existence of $C_{0}\left(R^{2}\right)$ solutions to difference equations as in Example 2.1 is really a special case of the theorems mentioned above. However, in explicit examples similar to the one in Example 2.1, we can employ Lemmas 2.2 and 2.3 to avoid the use of a smooth mass density vanishing near the endpoints of integration. Also, the estimate given by Lemma 2.2 when it applies will be stronger than the general ones.

There are two questions left unresolved by these examples of $C_{0}\left(R^{n}\right)$ or $L_{p}\left(R^{n}\right)$ solutions to difference equations. Suppose we take a particular difference equation in $R^{n}$ for $n \geqslant 2$ and there is a $C_{0}\left(R^{n}\right)$ solution. What is the infimum of all $p>2$ for which there is a nonzero solution to the difference equation in $L_{p}\left(R^{n}\right)$ ? Also, if we fix a dimension $n \geqslant 2$, what is the infimum $\beta_{n}$ of all those $p>2$ for which there is a nonzero function in $L_{p}\left(R^{n}\right)$ with linearly dependent translates? The examples above show $2 \leqslant \beta_{n} \leqslant$ $2 n /(n-1)$. The next theorem will help to answer these questions. Notice that if $f \in L_{p}\left(R^{n}\right)$ or $f \in C_{0}\left(R^{n}\right)$ and for some difference operator $D$, $D f=0$, then taking the Fourier transform of $D f$ as a tempered distribution shows that $f$ as a tempered distribution is supported in the zero set $\mathscr{I}_{p}$ of the characteristic trigonometric polynomial. For the relevant background material see [4], [15], [18], [22], [25], [30].

THEOREM 2.6. If $S \subset R^{n}$ is a closed subset of Hausdorff dimension no larger than $n-1$, and $T \neq 0$ is a tempered distribution with $\operatorname{supp}(T) \subset S$ such that $\hat{T} \in L_{p}\left(R^{n}\right)$, then $p \geqslant 2 n /(n-1)$.

Proof. By multiplying $T$ with a $C^{\infty}$-function of compact support, one may assume that $\hat{T}$ is bounded and $S$ is compact. Then for $0<\alpha<n$, the integral

$$
I=\int_{R^{n}}|\hat{T}(x)|^{2}|x|^{\alpha-n} d x<\infty
$$

if and only if the integral

$$
J=\int_{|x|>1}|\hat{T}(x)|^{2}|x|^{\alpha-n} d x<\infty
$$

Assume $\infty>p>2$ and write $p=2 r$ with $1<r<\infty$; let $1 / r+1 / q=1$. Then by Hölder's inequality,

$$
J \leqslant\left(\int_{|x|>1}|\hat{T}(x)|^{p} d x\right)^{1 / r}\left(\int_{|x|>1}|x|^{(\alpha-n) q} d x\right)^{1 / q} .
$$


Hence, since $\hat{T} \in L_{p}\left(R^{n}\right), J<\infty$ if

$$
\int_{|x|>1}|x|^{(\alpha-n) q} d x<\infty
$$

That is, $I<\infty$ if $(\alpha-n) q+n<0$. By a theorem of Deny [3, p. 142], at least for $n-1<\alpha<n$, if $I<\infty$, then there exists a probability measure $\mu$ with $\operatorname{supp}(\mu) \subset \operatorname{supp}(T)$ such that

$$
\int_{R^{n}}|\hat{\mu}(x)|^{2}|x|^{\alpha-n} d x<\infty .
$$

But by a theorem of Frostman [8, p. 90], the Hausdorff dimension of $S$ is also the capacitary dimension of $S$. Hence, for $\alpha>n-1$, there exists no probability measure $\mu$ with $\operatorname{supp}(\mu) \subset S$ and

$$
\int_{R^{n}}|\hat{\mu}(x)|^{2}|x|^{\alpha-n} d x<\infty .
$$

Therefore, for all $\alpha>n-1$, we must have $(\alpha-n) q+n \geqslant 0$. That is,

$$
(n-\alpha) / n \leqslant 1 / q=(r-1) / r=(p-2) / p
$$

for all $\alpha>(n-1)$. Letting $\alpha \downarrow(n-1)$ gives $1 / n \leqslant(p-2) / p$ and so $p \geqslant$ $2 n /(n-1)$.

We can see from this theorem that the $L_{p}$ estimates in Examples 2.1 and 2.4 are the best possible because the zero set of a nontrivial trigonometric polynomial has Hausdorff dimension equal to some integer $1,2, \ldots, n-1$.

CoROllary 2.7. Any nonzero function $f \in L_{p}\left(R^{n}\right)$ with $p<2 n /(n-1)$ has linearly independent translates.

Proof. The zero set of a nonzero trigonometric polynomial $p$ on $R^{n}$ is the intersection with $R^{n}$ of an analytic variety in $C^{n}$. By theorems in Whitney [33] and [35], see also Whitney and Bruhat [34], the zero set of $p$ is a locally finite union of analytic manifolds. By Lemma 1.1, the Hausdorff dimension of this manifold is never equal to $n$. Hence, the Hausdorff dimension of the zero set of $p$ is no larger than $n-1$.

It is not clear whether functions in $L_{p}\left(R^{n}\right)$ with $p=2 n /(n-1)$ and $n \geqslant 2$ also have independent translates and it would be interesting to be able to resolve this case for the critical value of $p$. Also, Professor J.-P. Kahane has shown in a letter by a different argument that if $S \subset R^{n}$ is a closed set with Hausdorff dimension $k<n$, and $T \neq 0$ is a tempered distribution with $\operatorname{supp}(T) \subset S$ and $\hat{T} \in L_{p}\left(R^{n}\right)$, then $p \geqslant 2 n / k$. This allows one to get better lower bounds for the $L_{p}$-class of a solution to a particular difference equation. It would be useful to know some trigonometric polynomials on $R^{n}$ with $k$-dimensional zero sets that are not locally finite unions of subsets of hyperplanes. 
In contrast with the examples so far presented in this section, some difference equations, although admitting nonzero solutions, do not have any solutions which vanish at infinity. In order to give examples of this type in $R^{n}$, some of the theory of distributions supported in a vector subspace $R^{h} \subset R^{n}$ will be needed. As in Schwartz [31, Tome I, pp. 100-102], represent the points $z \in R^{n}$ as $z=(x, y)$ with $x \in R^{h}, y \in R^{k}$ and $h+k=n$. The restriction $\varphi$ on $R^{h}$ of a $C^{\infty}$-function $\bar{\varphi}(x, y)$ defined on $R^{n}$ with compact support is defined by $\varphi(x)=\bar{\varphi}(x, 0)$ for all $x \in R^{h}$; the extension $\bar{T}$ to $R^{n}$ of a distribution $T$ on $R^{h}$ is defined, for $C^{\infty}$-functions $\bar{\varphi}$ on $R^{n}$ with compact support, by $\bar{T}(\bar{\varphi})=T(\varphi)$. A derivative transverse to the subspace $R^{h}$ is any multi-indexed partial derivative with respect to the component variables of $y$.

THEOREM 2.8. All distributions $T_{(x, y)}$ with support contained in a subspace $R^{h}$ of $R^{n}$ admit a unique decomposition as a locally finite linear combination of derivatives transverse to $R^{h}$ of extensions to $R^{n}$ of distributions defined on $R^{h}$ :

$$
T_{(x, y)}=\sum_{q} D_{y}^{q}\left(\bar{T}_{q}\right)_{(x, y)}
$$

Each of the distributions $T_{q}$ has support contained in the support of $T$ and the distributions $T_{q}$ depend continuously on $T$.

If the support of the distribution $T$ is compact, then this sum reduces to a finite sum; a particular case of this theorem is that a distribution which is supported at a point is a finite sum of derivatives of the Dirac mass at the point.

THEOREM 2.9. Suppose $D$ is a difference operator on $R^{n}$ for which the characteristic zero set $\mathscr{Z}_{p}$ is a locally finite union of subsets of hyperplanes in $R^{n}$. Then $D f=0$ has no nonzero solutions $f \in C_{0}\left(R^{n}\right)$ or in $L_{p}\left(R^{n}\right)$ for $1 \leqslant p<\infty$.

Proof. Suppose $D f=0$ with $0 \neq f \in L_{p}\left(R^{n}\right)$ and $1 \leqslant p<\infty$. Let $\varphi$ be a continuous function with compact support in $R^{n}$ such that the convolution $f * \varphi \neq 0$. Then $D f * \varphi=D(f * \varphi)$ and, hence, $0=D(f * \varphi)$ also. But $f * \varphi$ is in $C_{0}\left(R^{n}\right)$, and so without loss of generality only the case $0 \neq f \in C_{0}\left(R^{n}\right)$ is considered. In addition, by convolving again with a $C^{\infty}$-function having compact support, we may assume $0 \neq f \in C_{0}\left(R^{n}\right)$ and $f$ is also a $C^{\infty}$. function.

If $D f=0$, then as indicated above, $\hat{f}$ has a support in $\mathscr{Z}_{p}$. By the assumption on the structure of $\mathscr{I}_{p}$, there exist a point $x \in \operatorname{supp} \hat{f}$ and an open set $U$ about $x$ such that $U \cap \operatorname{supp} \hat{f}$ is contained in a hyperplane $H \subset R^{n}$. Let $\varphi$ be a $C^{\infty}$-function with compact support in $U$ which is identically one in a neighborhood of $x$; then, because $\varphi$ is the Fourier transform of an integrable function, $\hat{f} \varphi$ is also the Fourier transform of a 
$C^{\infty}$-function $F$ in $C_{0}\left(R^{n}\right)$. By applying a rotation to $F$ and then multiplying the resulting function by a character $\gamma(x)=\exp (i t \cdot x)$ for $t \in R^{n}$, we may assume $\hat{F}$ is supported in the subspace $R^{n-1}=\left\{\left(x_{1}, \ldots, x_{n-1}, 0\right): x_{i} \in R\right\}$ of $R^{n}$ without altering the fact that $0 \neq F \in C_{0}^{\infty}\left(R^{n}\right)$.

But now Theorem 2.8 says that $\hat{F}=\Sigma_{q} D_{y}^{q}\left(\bar{T}_{q}\right)_{(x, y)}$ where $x \in R^{n-1}, y \in$ $R$, and the sum is a finite sum of derivatives with respect to $y$. Let $N$ be fixed with $N-1$ the largest value of $q$ in the sum and let $\varphi$ be an arbitrary $C^{\infty}$-function on $R^{n}$ with compact support. Then the partial derivative $\left(\partial^{N} / \partial y^{N}\right) F$ is a distribution with

$$
\begin{aligned}
\left(\frac{\partial^{N}}{\partial y^{N}} F\right)^{\wedge}(\varphi) & =\left(\frac{\partial^{N}}{\partial y^{N}} F\right)(\hat{\varphi})=(-1)^{N} F\left(\frac{\partial^{N}}{\partial y^{N}} \hat{\varphi}\right) \\
& =(-i)^{N} F\left(\left(y^{N} \varphi\right)^{\wedge}\right)=(-i)^{N} \hat{F}\left(y^{N} \varphi\right) .
\end{aligned}
$$

But for any $q$,

$$
D_{y}^{q}\left(\bar{T}_{q}\right)_{(x, y)}\left(y^{N} \varphi\right)=(-1)^{q}\left(\bar{T}_{q}\right)_{(x, y)}\left(D_{y}^{q}\left(y^{N} \varphi\right)\right)=(-1)^{q}\left(T_{q}\right)_{x}(\Psi)
$$

where $\Psi(x)=D_{y}^{q}\left(y^{N} \varphi\right)(x, 0)$. Since $N>q$ for any $q$ in the sum representing $\hat{F}, \Psi$ is identically zero and, therefore, the term $D_{y}^{q}\left(\bar{T}_{q}\right)_{(x, y)}\left(y^{N} \varphi\right)=0$. Hence, $\left(\partial^{N} / \partial y^{N}\right) F$ is zero because its Fourier transform is zero when evaluated on an arbitrary test function. This means that as a function of the variable $y, F$ is a polynomial of degree at most $N-1$ for each fixed value for the variables $x_{1}, \ldots, x_{n-1}$. This is impossible because $0 \neq F \in C_{0}\left(R^{n}\right)$.

EXAMPLE 2.10. If instead of the difference equation in Example 2.1, one considers the difference equation

$$
4 f(x, y)=f(x+1, y)+f(x-1, y)+f(x, y+1)+f(x, y-1),
$$

then the corresponding polynomial $p(x, y)=4(2-\cos x-\cos y)$ and $\mathscr{I}_{p}$ is a discrete set. Theorem 2.9 shows that this difference equation has no solutions in $L_{p}\left(R^{n}\right)$ for $1 \leqslant p<\infty$ or in $C_{0}\left(R^{n}\right)$ other than zero.

The next corollary was communicated in a letter by Professor $R$. E. Edwards; he used an argument which suggested the proof of Theorem 2.9.

CoRollaRY 2.11. Any nonzero function in $C_{0}(R)$ or $L_{p}(R)$ for $1 \leqslant p<\infty$ has linearly independent translates.

Proof. A trigonometric polynomial on the real line can be extended to an analytic function of the complex plane; so it must have a discrete set of zeros unless it is identically zero. Hence, any difference equation $D f=0$ with $D$ not identically zero must have the characteristic zero set $\mathfrak{Z}_{p}$ discrete; this means that Theorem 2.9 applies to any nontrivial difference equation on the real line. 
The examples given in the beginning of this section and Theorem 2.9 suggest a characterization of those difference equations over $R^{n}$ for $n \geqslant 2$ which have a nonzero solution in $C_{0}\left(R^{n}\right)$. To do this, we need to use Theorem 1 in Baggett and Taylor [36] which implies that an analytic submanifold of $R^{n}$ which does not lie in any hyperplane must support many functions with Fourier transform in $C_{0}\left(R^{n}\right)$. Using this, the same decomposition theorem of Whitney used in Corollary 2.7 gives the following theorem.

THEOREM 2.12. A difference equation over $R^{n}$ for $n \geqslant 2$ has no nonzero solutions in $C_{0}\left(R^{n}\right)$ if and only if the zero set of the characteristic trigonometric polynomial is a locally finite union of subsets of hyperplanes in $R^{n}$.

It is perhaps worth pointing out that many of the facts about the $L_{p}$-class of solutions to linear difference equations which are mentioned in this section also apply to solutions to partial differential equations on $R^{n}$. For example, any nonzero function $f \in L_{p}\left(R^{n}\right)$ with $p<2 n /(n-1)$ cannot be a solution to a nontrivial partial differential equation on $R^{n}$. Also, parallel to Examples 2.1 and 2.4, there are nontrivial partial differential equations on $R^{n}$ for $n \geqslant 2$ which admit $C_{\infty}$-solutions that are in $C_{0}\left(R^{n}\right)$ and in all $L_{p}\left(R^{n}\right)$ for $p>2 n /(n$ $-1)$. In proving these facts, the trigonometric polynomials are replaced by real polynomials of several variables and the theory of tempered distributions is used analogously.

3. Compact abelian groups. In compact abelian groups, there are always nonzero functions in $L_{1}(G)$ which have linearly dependent translates; however, if $f \in L_{1}(G)$ and the Fourier transform $\hat{f}$ is never zero, then $f$ has linearly independent translates. It is natural to try to characterize those functions which do have a dependence relation among the translates. It seems difficult to get a simple characterization of this phenomenon in general as a subsequent example will show, but in some special cases this can be done. For example, here is an instance of a theorem of Lech [19] which can be used to solve this problem for the circle group $T$. We thank Professor B. Baishanski for pointing out this theorem; it would be enlightening to know a simple proof.

THeOREM 3.1. Suppose that $c_{1}, \ldots, c_{n} \in C$ and nonzero $t_{1}, \ldots, t_{n} \in C$. Consider the integers $k$ such that $\sum_{j=1}^{n} c_{j} t_{j}^{k}=0$. If this set of integers is infinite, then it consists of a finite number of the residue classes in $Z$ modulo some whole number $r$ and, in addition, at most a finite number of other integers.

Remarks. This theorem is proved by Lech in greater generality than is stated here; the theorem will be used in the case that the scalars $t_{i}$ have $\left|t_{i}\right|=1$. It is not hard to see that when the $\left(t_{i}\right)$ are free abelian generators in the circle group, Kronecker's lemma and Lech's theorem show that there is at 
most a finite number of solutions $k \in Z$ to an equation of the form $\sum_{j=1}^{n} c_{j} t_{j}^{k}$ $=0$, unless all the coefficients $c_{j}=0$. What is the number of such solutions? Is it less than $n$ ? This is tantamount to asking whether when $\left(t_{i}\right)$ are free abelian generators in the circle group and $s_{1}, \ldots, s_{n}$ are distinct integers, is it the case that $\operatorname{det}\left(t_{j}^{s_{i}}\right) \neq 0$ ? This problem has been solved in a few special cases (see [32]).

THEOREM 3.2. A function $f \in L_{p}(T)$ or $f \in C(T)$ has a nontrivial dependence relation among its translates if and only if $f(z)$ has the form

$$
f(z)=p(z, \bar{z})+\sum_{j=1}^{k} z^{r} f_{j}\left(z^{r}\right)
$$

where $p(z, \bar{z})$ is a polynomial with complex coefficients, $r \geqslant 2$ is an integer, each $r_{j}$ is an integer and $k \leqslant r-1$, and each function $f_{j} \in L_{p}(T)$ or $f_{j} \in C(T)$ as $f \in L_{p}(T)$ or $f \in C(T)$. That is, $f$ has linearly independent translates if and only if the support of its Fourier transform $\hat{f}$ on $Z$ contains a complete set of residues modulo every nonzero integer.

Proof. Assume that for distinct $t_{1}, \ldots, t_{n} \in T$ and some scalars $c_{1}, \ldots, c_{n}$ $\in C$, one has $\sum_{j=1}^{n} c_{j} T_{t} f=0$. Taking the Fourier transform shows that the support of $f$ is contained in $\left\{k \in Z: \sum_{j=1}^{n} c_{j} t_{j}^{k}=0\right\}$. If one assumes that not all of the scalars $c_{j}$ are zero, then this set cannot be all of $Z$. Using Theorem 3.1 , the support of $\hat{f}$ is contained in a proper number of the residue classes modulo $r$ for some $r \geqslant 2$ excepting a finite number of terms; so there exists a polynomial $p(z, \bar{z})$ such that $f-p$ has spectrum in a union of residue classes $r_{j} \bmod r$. But then in any of the Banach spaces $L_{p}(T)$ or $C(T)$, there exists a projection $Q_{j}$ with range the functions having spectrum $\left\{k \in Z: k=r_{j}\right.$ $\bmod r\} ; Q_{j}$ is given by

$$
Q_{j}(f)(z)=\sum_{l=0}^{r-1} \frac{\bar{s}^{-l r_{j}}\left(s^{l} z\right)}{r}
$$

where $s$ is any fixed primitive $r$ th-root of unity. Hence, as $f \in L_{p}(T)$ or $f \in C(T)$, the function

$$
f(z)-p(z, \bar{z})=\sum_{j=1}^{k} z^{r} f_{j}\left(z^{r}\right)
$$

for some functions $f_{j} \in L_{p}(T)$ or $f_{j} \in C(T)$.

Conversely, suppose $f$ has the form above and let $s$ be a primitive rth-root of unity. Define $P_{1}$ to be the difference operator $\Pi_{j=1}^{k}\left(T_{s}-s^{r_{j} I}\right)$. Then since $\left(T_{s}-s^{r_{j} I}\right)\left(z^{r_{j}} f_{j}\left(z^{r}\right)\right)=0$,

$$
P_{1}\left(\sum_{j=1}^{k} z^{r} f_{j}\left(z^{r}\right)\right)=0 .
$$


Also, if $P_{2}$ is the difference operator $\Pi_{l=-M}^{M}\left(T_{t}-t^{l} I\right)$ where $M \geqslant \operatorname{deg}(p)$, then $P_{2}(p)=0$. Hence, for any such $P_{1}$ and $P_{2}$, we have $\left(P_{1} P_{2}\right) f=0$. By taking $t$ with an irrational argument, one is guaranteed that $P_{1} P_{2}$ is not an identically zero operator and so $\left(P_{1} P_{2}\right) f=0$ represents a nontrivial difference equation that $f$ satisfies.

COROLLARY 3.3. The only functions in $L_{1}(T)$ which have a linear dependence relation among the translates by free abelian generators in $T$ are the trigonometric polynomials.

Proof. See the Remarks after Theorem 3.1 and the proof of Theorem 3.2.

The form of a function $f \in L_{\infty}(T)$ which satisfies a nontrivial difference equation is that of a proper sum of characters multiplied by periodic functions. It seems unlikely that this will remain true for the higher dimensional tori too, but it certainly cannot be generalized to all connected compact' metric abelian groups as the following example shows. This theorem also shows how badly the same structure theorem may fail if $T$ is replaced by $R$.

EXAMPle 3.4. Consider the function $p(x)=\sin x-\sqrt{2} \sin 2 \pi x$. This function has an infinite number of zeros in $R$ but its zero set does not contain an infinite arithmetic sequence. First, notice that when the fractional part $\{x\} \in\left(\frac{1}{8}, \frac{3}{8}\right)$, then $p(x)<0$; and when $\{x\} \in\left(\frac{5}{8}, \frac{7}{8}\right)$, then $p(x)>0$. So $p$ has infinitely many zeros and also no infinite arithmetic sequence $\left(x_{0}+r j\right.$ : $j \geqslant 0)$ can all be zeros of $p(x)$ unless $r$ is rational. Suppose that $\left(x_{0}+r j\right.$ : $j \geqslant 0$ ) are zeros of $p$ and $r=a / b$ with $a$ and $b$ relatively prime integers, $a \neq 0$ and $b>0$. Let $j=0,2 b, b$ to get

$$
\sin x_{0}=\sqrt{2} \sin 2 \pi x_{0}, \quad \sin \left(x_{0}+2 a\right)=\sqrt{2} \sin 2 \pi\left(x_{0}+2 a\right),
$$

and

$$
\sin \left(x_{0}+a\right)=\sqrt{2} \sin 2 \pi\left(x_{0}+a\right)
$$

Then

$$
\sin \left(x_{0}+2 a\right)=\sqrt{2} \sin \left(2 \pi x_{0}+4 \pi a\right)=\sqrt{2} \sin 2 \pi x_{0}=\sin x_{0} .
$$

Hence, there exists $k_{0} \in Z$ with

$$
x_{0}+2 a=x_{0}+2 \pi k_{0} \text { or } x_{0}+2 a=\pi-x_{0}+2 \pi k_{0} \text {. }
$$

The first case says that $\pi$ is rational and so we must have $x_{0}+a=\pi\left(k_{0}\right.$ $\left.+\frac{1}{2}\right)$. Hence,

$$
\begin{aligned}
\pm 1 & =\sin \pi\left(k_{0}+\frac{1}{2}\right)=\sin \left(x_{0}+a\right) \\
& =\sqrt{2} \sin 2 \pi\left(x_{0}+a\right)=\sqrt{2} \sin 2 \pi^{2}\left(k_{0}+\frac{1}{2}\right)
\end{aligned}
$$


This means $\sin 2 \pi^{2}\left(k_{0}+\frac{1}{2}\right)= \pm 1 / \sqrt{2}$ and so there exists $k_{1} \in Z$ such that $2 \pi^{2}\left(k_{0}+\left(\frac{1}{2}\right)\right)=\pi / 4+k_{1} \pi / 2$. This again forces $\pi$ to be rational. Hence, $p$ cannot have an infinite arithmetic progression in its set of zeros.

Now enumerate the zeros of $p$ as $\left(x_{n}\right)$ and let $\left(a_{n}\right)$ be nonzero scalars with $\sum_{n=1}^{\infty}\left|a_{n}\right|<\infty$. Consider the function $F: R \rightarrow C$ given by $F(x)=$ $\sum_{n=1}^{\infty} a_{n} \exp \left(i x_{n} x\right)$. Then $F$ is an almost periodic function with Fourier transform having support equal to the zeros of $p$. Because

$$
2 i p(x)=\exp (i x)-\exp (-i x)-\sqrt{2} \exp (2 \pi i x)+\sqrt{2} \exp (-2 \pi i x),
$$

the function $F$ satisfies the difference equation

$$
0=T_{1} F-T_{-1} F-\sqrt{2} T_{2 \pi} F+\sqrt{2} T_{-2 \pi} F .
$$

Because the spectrum of $F$ is infinite but contains no infinite arithmetic sequence, $F$ cannot be written as a sum of periodic functions on $R$ multiplied by characters of $R$. This makes it clear that there cannot be a theorem exactly like Theorem 3.2 which characterizes the functions in $L_{\infty}(R)$ that satisfy a difference equation. However, it is the case that any uniformly continuous bounded function which satisfies a nontrivial difference equation is almost periodic (see Kahane [14, p. 43]); this fact also gives an alternate method of proving Corollary 2.9 because nonzero functions in $C_{0}(R)$ are not almost periodic.

Example 3.4 can also be induced onto a connected compact abelian group in this manner. Let $X$ be the subgroup of $R$ generated by the sequence $\left(x_{n}\right)$ of zeros of the trigonometric polynomial $p$. In the discrete topology, $X$ is the dual group of a connected compact metric abelian group $G$ and the point evaluations $\gamma_{n}: G \rightarrow T$ given by $\gamma_{n}(g)=x_{n}(g)$ are continuous characters on $G$. Let $F(g)=\sum_{n=1}^{\infty} a_{n} \gamma_{n}(g)$; this defines a continuous function $F: G \rightarrow C$ which is not a finite sum of characters on $G$ multiplied by periodic functions. Also, the character on $X$ given by restricting $x \rightarrow \exp (i K x)$ to $X$ defines an element $R_{K}$ of $G$. By taking Fourier transforms, one can see that $F$ satisfies the difference equation

$$
0=T_{R_{1}} F-T_{R_{-1}} F-\sqrt{2} T_{R_{2 \pi}} F+\sqrt{2} T_{R_{-2 \pi}} F .
$$

This example shows that there will not be a simple theorem like Theorem 3.2 which characterizes the continuous functions on connected compact metric abelian groups that have a dependence relation among their translates. It would be worthwhile to know if there is a similar example on the torus $T^{2}$.

\section{REFERENCES}

1. P. Batchelder, An introduction to linear difference equations, Dover, New York, 1967.

2. N. Chako, Asymptotic expansions of double and multiple integrals occurring in diffraction theory, J. Inst. Math. Appl. 1 (1965), 372-422.

3. J. Deny, Les potentiels d'energie finie, Acta Math. 82 (1950), 107-183. 
4. W. Donoghue, Distributions and Fourier transforms, Academic Press, New York, 1969.

5. R. E. Edwards, Fourier series, vols. 1 and 2, Holt, Rinehart, and Winston, New York, 1967.

6. __ Functions whose translates are independent, Ann. Inst. Fourier (Grenoble) 2 (1950), 31-72.

7. A. Erdélyi, Asymptotic expansions, Dover, New York, 1956.

8. O. Frostman, Potentiel d'équilibre et capacité des ensembles, Lunds Universitets Mathematiske Seminarium Band 2 (1935), 1-118.

9. S. Goldberg, Introduction to difference equations, Wiley, New York, 1958.

10. C. S. Herz, Fourier transforms related to convex sets, Ann. of Math. (2) 75 (1962), 81-92.

11. E. Hewitt and K. Ross, Abstract harmonic analysis, vols. 1 and 2, Springer-Verlag, New York, 1963.

12. A. and C. Ionescu-Tulcea, On the existence of a lifting commuting with the left translations of an arbitrary locally compact group, Fifth Berkeley Symposium on Math. Statistics and Probability, vol. 2, part 1, Univ. California Press, Berkeley, 1965, pp. 63-97.

13. J. Jenkins, $A$ fixed point theorem for exponentially bounded groups, J. Functional Analysis 22 (1976), 346-353.

14. J.-P. Kahane, Lectures on mean periodic functions, Tata Institute, Bombay, 1959.

15. __ Some random series of functions, D. C. Heath, Lexington, Mass., 1968.

16. J. H. Kemperman, A general functional equation, Trans. Amer. Math. Soc. 86 (1957), $28-56$.

17. L. Kuipers and H. Niederreiter, Uniform distribution of sequences, Wiley, New York, 1974.

18. N. Landkof, Foundations of modern potential theory, Springer-Verlag, New York, 1972.

19. C. Lech, A note on recurring series, Ark. Mat. 2 (1953), 417-421.

20. W. Littman, Fourier transforms of surface carried measures and differentiability of surface averages, Bull. Amer. Math. Soc. 69 (1963), 766-770.

21. J. López and K. Ross, Sidon sets, Dekker, New York, 1975.

22. B. Malgrange, Existence et approximation des solutions des équations aux derivées partielles et des équations de convolution, Ann. Inst. Fourier (Grenoble) 6 (1955-56), 271-355.

23. Y. Meyer, Comportment asymptotique des solutions de certaines équations de convolution, $\mathrm{J}$. Math. Pures et Appl. 55 (1976), 69-98.

24. D. Montgomery and L. Zippin, Topological transformation groups, Interscience Publishers, New York, 1966.

25. J. Riss, Eléments de calcul différential et théorie des distributions sur les groupes abélians localement compacts, Acta Math. 89 (1953), 45-105.

26. J. Rosenblatt, Equivalent invariant measures, Israel J. Math. (1974), 261-270.

27. K. Ross, Sur les compacts associés à un ensemble de Sidon, C. R. Acad. Sci. Paris 275A (1972), A183-A185.

28. , Fatou-Zygmund sets, Proc. Cambridge Philos. Soc. 73 (1973), 57-65.

29. W. Rudin, Fourier analysis on groups, Interscience Publishers, New York, 1962.

30. L. Schwartz, Etude des sommes d'éxponentielles réelles; Actualités Sci. Indust., no. 959, Hermann, Paris, 1943.

31. _. Théorie des distributions, Tomes I, II, Hermann, Paris 1957, 1959.

32. H. S. Shapiro, A vanishing determinant, Amer. Math. Monthly 69 (1962), 929-930.

33. H. Whitney, Tangents to an analytic variety, Ann. of Math. 81 (1965), 496-549.

34. H. Whitney and F. Bruhat, Quelques propriétés fondamentales des ensembles analytiquesréels, Comment. Math. Helv. 33 (1959), 132-160.

35. H. Whitney, Local properties of analytic varieties, Differential and Combinatorial Topology, Ed., S. Cairns, Princeton Univ. Press, Princeton, N. J., 1965.

36. Larry Baggett and Keith Taylor, Riemann-Lebesgue subsets of $R^{n}$ and representations which vanish at infinity, J. Functional Analysis 28 (1978), 168-181.

Department of Mathematics, Oho State University, Columbus, Oho 43210 